PROCEEDINGS OF THE

AMERICAN MATHEMATICAL SOCIETY

Volume 132, Number 1, Pages 87-95

S 0002-9939(03)07239-3

Article electronically published on August 21, 2003

\title{
TOPOLOGICAL SPECTRUM OF LOCALLY COMPACT CANTOR MINIMAL SYSTEMS
}

HIROKI MATUI

(Communicated by Michael Handel)

\begin{abstract}
We show that there exists a locally compact Cantor minimal system whose topological spectrum has a given Hausdorff dimension.
\end{abstract}

\section{INTRODUCTION}

The purpose of this paper is to introduce the topological spectrum for LCCM systems and to discuss the relation to the dimension groups. In the measurable setting, the group of $L^{\infty}$-eigenvalues of a nonsingular ergodic transformation were studied by several authors ( $\underline{\mathrm{A}}, \underline{\mathrm{AN}}],[\mathrm{IKS},[\mathrm{HMP}]$ ), and it was known that the group may be uncountable and can have arbitrary Hausdorff dimension. We would like to consider its topological analogue in this paper.

Our object of study is an LCCM or CM system. Let us introduce them. A homeomorphism $\phi$ from a topological space $X$ to itself is called minimal, if every $\phi$-orbit is dense in $X$. This is obviously equivalent to nonexistence of nontrivial closed invariant sets. When $X$ is the Cantor set and $\phi \in \operatorname{Homeo}(X)$ is minimal, we say that $(X, \phi)$ is a Cantor minimal system, or CM system for short. A non-closed open subset of the Cantor set is called a locally compact Cantor set. When $\phi$ is a minimal homeomorphism on the locally compact Cantor set $X$, the pair $(X, \phi)$ is called a locally compact Cantor minimal system, or LCCM system for short. CM and LCCM systems were studied in many papers (GPS, D, [M]), and it was proved that their dimension groups are the complete invariant for topological orbit equivalence. We view these topological dynamical systems as an analogue of ergodic systems of type $\mathrm{II}_{1}$ or $\mathrm{II}_{\infty}$.

At first we give a definition of eigenvalues and eigenfunctions. Of course the eigenfunctions have to be continuous, and we will see that this restriction implies the richness of continuous characters. In Section 2, after introducing the Polish topology on the topological spectrum group, we will consider the topology of the dual group and duality. In Section 3, we will construct an LCCM system whose topological spectrum has a given Hausdorff dimension and which is strong orbit equivalent to a given LCCM system. The method of construction is similar to that of measurable cases, given in $[\mathrm{A}$.

Received by the editors April 12, 2002.

2000 Mathematics Subject Classification. Primary 37B05.

(C)2003 American Mathematical Society 


\section{TOPOLOGICAL SPECTRUM}

We begin with the definition of topological spectrum.

Definition 1. Let $(X, \phi)$ be a CM or LCCM system. If a continuous function $f: X \rightarrow \mathbb{R} / \mathbb{Z}$ and $\lambda \in \mathbb{R} / \mathbb{Z}$ satisfy $f \phi=f+\lambda$, we call $f$ an eigenfunction and $\lambda$ an eigenvalue of $(X, \phi)$. We denote the set of eigenfunctions by $E(\phi)$ and the set of eigenvalues by $e(\phi)$. Each of these sets clearly forms an abelian group. We call $e(\phi)$ the topological spectrum of $(X, \phi)$.

We denote by $P$ the canonical homomorphism from $E(\phi)$ to $e(\phi)$. Equip $E(\phi)$ with the topology of uniform convergence on compact subsets on $X$ and $e(\phi)$ with the quotient topology. For $\lambda \in \mathbb{R} / \mathbb{Z}$ we write the distance from zero by $|\lambda|$.

Lemma 2. Let $(X, \phi)$ be a $C M$ or LCCM system and let $U \subset X$ be a compact open set with $U \cap \phi(U) \neq \emptyset$. Then, $d(f, g)=\max \{|f(x)-g(x)| ; x \in U\}$ gives a complete metric on $E(\phi)$, and $E(\phi)$ is a Polish group.

Proof. For every compact set $K \subset X$, there exists $N$ such that $K \subset \bigcup_{|n| \leq N} \phi^{n}(U)$. Therefore $d(\cdot, \cdot)$ gives a metric. Separability and completeness follow from those properties of $C(U)$.

The following lemma is obvious.

Lemma 3. For a CM or LCCM system $(X, \phi)$, we have the following.

(i) The canonical inclusion $e(\phi) \rightarrow \mathbb{R} / \mathbb{Z}$ is continuous.

(ii) For every $x \in X$ there exists a continuous homomorphism $P_{x}: e(\phi) \rightarrow$ $E(\phi)$ such that $P P_{x}=i d$ and $P_{x}(\lambda)(x)=0$ for all $\lambda \in e(\phi)$. Especially, $E(\phi) \cong e(\phi) \times \mathbb{R} / \mathbb{Z}$ as topological groups.

(iii) The topological spectrum $e(\phi)$ is a Polish group.

Since $e(\phi)$ is Polish, a Borel set of $e(\phi)$ is Borel in $\mathbb{R} / \mathbb{Z}$ (see $[S$, Theorem 4.5.4]). In the same fashion as in the measurable case $\mathrm{AN}$, we can show that a Polish topology on $e(\phi)$ under which the injection map $e(\phi) \rightarrow \mathbb{R} / \mathbb{Z}$ is continuous is unique. In fact, if $N_{1}$ and $N_{2}$ are Polish topologies under which the injection map $e(\phi) \rightarrow \mathbb{R} / \mathbb{Z}$ is continuous, then the Borel structure generated by $N_{1}$ agrees with the Borel structure inherited from $\mathbb{R} / \mathbb{Z}$, which in turn is the Borel structure generated by $N_{2}$. Hence [S, Proposition 3.5.8] tells us that the identity map from $\left(e(\phi), N_{1}\right)$ to $\left(e(\phi), N_{2}\right)$ is continuous and two topologies agree. Note that, however, (ii) of the above lemma is rather different from the measurable case [AN, Theorem 2.5]. Fix $x_{0} \in X$ and $P_{x_{0}}: e(\phi) \rightarrow E(\phi)$. For every $x \in X$,

$$
e(\phi) \ni \lambda \mapsto P_{x_{0}}(\lambda)(x) \in \mathbb{R} / \mathbb{Z}
$$

gives a (continuous) character of $e(\phi)$. We denote it by $\tau_{x}$ and the set of all characters of $e(\phi)$ by $\varepsilon(\phi)$. In contrast to the measurable case, the splitting homomorphism $P_{x_{0}}$ ensures the rich structure of $\varepsilon(\phi)$, unless $e(\phi)$ is trivial.

Lemma 4. The spectrum e $(\phi)$ of a CM or LCCM system $(X, \phi)$ is a proper subset of $\mathbb{R} / \mathbb{Z}$. Moreover, e $(\phi)$ is a weak Dirichlet set of $\mathbb{R} / \mathbb{Z}$.

Proof. Take a probability measure $\mu$ on $e(\phi)$. For $x_{0} \in X$, there exists $\left\{a_{n}\right\}_{n} \subset$ $\mathbb{Z} \backslash\{0\}$ such that $x_{n}=\phi^{a_{n}}\left(x_{0}\right) \rightarrow x_{0}$. By the Lebesgue convergence theorem, we have

$$
\lim _{n \rightarrow \infty} \int_{e(\phi)}\left|e^{2 \pi i a_{n} \lambda}-1\right|^{2} d \mu=\lim _{n \rightarrow \infty} \int_{e(\phi)}\left|e^{2 \pi i \tau_{x_{n}}(\lambda)}-e^{2 \pi i \tau_{x_{0}}(\lambda)}\right|^{2} d \mu=0
$$


that is, $e(\phi)$ is a weak Dirichlet set.

If $(X, \phi)$ has a finite invariant measure, it is not hard to see that $e(\phi)$ is at most countable and endowed with the discrete topology. Hence we will mainly focus our attention on the case that $(X, \phi)$ is an LCCM system without finite invariant measures.

A Bratteli-Vershik model is the most convenient tool for the study of LCCM systems. We would like to review the notion of almost simple properly ordered Bratteli diagrams.

Definition 5. We say that $B=(V, E)$ is a Bratteli diagram when $V=\bigcup_{n=0}^{\infty} V_{n}$ and $E=\bigcup_{n=1}^{\infty} E_{n}$ are disjoint unions of finite sets of vertices and edges with source maps $s: E_{n} \rightarrow V_{n-1}$ and range maps $r: E_{n} \rightarrow V_{n}$ both of which are surjective. We always assume that $V_{0}$ consists of one point $v_{0}$. For a Bratteli diagram $B=(V, E)$,

$$
X_{B}=\left\{\left(e_{n}\right)_{n} ; e_{n} \in E_{n}, r\left(e_{n}\right)=s\left(e_{n+1}\right) \text { for all } n \in \mathbb{N}\right\}
$$

is called the infinite path space of $B$. The space $X_{B}$ is endowed with the natural product topology.

The following definition can be found in $[\mathrm{D}]$ and $[\mathrm{M}]$.

Definition 6. Let $B=(V, E)$ be a Bratteli diagram.

(i) We say that $B=(V, E)$ is simple when for every $v \in V_{n}$ there exists $m>n$ such that all vertices of $V_{m}$ are connected to $v$.

(ii) Let us assume that there is an infinite path $\left(e_{n}\right)_{n} \in X_{B}$ such that $r^{-1}\left(r\left(e_{n}\right)\right)$ $=\left\{e_{n}\right\}$ for all $n \in \mathbb{N}$. The diagram $B$ is said to be almost simple when for every $v \in V_{n} \backslash\left\{r\left(e_{n}\right)\right\}$ and $v^{\prime} \in V_{n}$ there exists $m>n$ such that all vertices of $V_{m}$ that are connected to $v$ are also connected to $v^{\prime}$. We denote the infinite path $\left(e_{n}\right)_{n}$ by $\infty_{B}$.

(iii) When the finite set $r^{-1}(v)$ has a linear order for each $v \in V$, we call $B$ an ordered Bratteli diagram. We define the set of maximal infinite paths and minimal infinite paths by

$$
X_{B}^{\max }=\left\{\left(e_{n}\right)_{n} \in X_{B} ; e_{n} \text { is maximum in } r^{-1}\left(r\left(e_{n}\right)\right)\right\}
$$

and

$$
X_{B}^{\min }=\left\{\left(e_{n}\right)_{n} \in X_{B} ; e_{n} \text { is minimum in } r^{-1}\left(r\left(e_{n}\right)\right)\right\} .
$$

(iv) A simple ordered Bratteli diagram $B=(V, E)$ is said to be properly ordered when each of $X_{B}^{\max }$ and $X_{B}^{\min }$ consists of one point.

(v) An almost simple ordered Bratteli diagram $B=(V, E)$ is said to be properly ordered when $X_{B}^{\max }=X_{B}^{\min }=\left\{\infty_{B}\right\}$.

If $B=(V, E)$ is a properly ordered almost simple Bratteli diagram, we can define the Bratteli-Vershik map $\phi_{B}$ on $X_{B}$ (see $[\mathrm{D}$ ). The following is the model theorem of LCCM systems.

Theorem 7 ([D, Theorem 3.3]).

(i) When $B=(V, E)$ is a properly ordered almost simple Bratteli diagram, $\left(X_{B} \backslash\right.$ $\left.\left\{\infty_{B}\right\}, \phi_{B}\right)$ is an LCCM system.

(ii) When $(X, \phi)$ is an LCCM system, there exists an almost simple properly ordered Bratteli diagram $B=(V, E)$ such that $(X, \phi)$ is isomorphic to $\left(X_{B} \backslash\right.$ $\left.\left\{\infty_{B}\right\}, \phi_{B}\right)$. 
Let $B=(V, E)$ be a properly ordered almost simple Bratteli diagram and $\infty_{B}=$ $\left(e_{n}\right)_{n}$. For convenience we assume that $r\left(s^{-1}\left(V_{n} \backslash\left\{r\left(e_{n}\right)\right\}\right)\right)$ equals $V_{n+1} \backslash\left\{r\left(e_{n+1}\right)\right\}$ for all $n \in \mathbb{N}$ (see [M] Remark 2.5]). We denote the ideal part of $B=(V, E)$ by $\tilde{B}=(\tilde{V}, \tilde{E})$, that is, $\tilde{B}$ is a subdiagram of $B$ with

$$
\widetilde{V}_{0}=\left\{v_{0}\right\}, \tilde{V}_{n}=V_{n} \backslash\left\{r\left(e_{n}\right)\right\}, \quad \widetilde{V}=\bigcup_{n} \widetilde{V}_{n}
$$

and

$$
\widetilde{E}_{n}=E_{n} \backslash s^{-1}\left(s\left(e_{n}\right)\right), \quad \widetilde{E}=\bigcup_{n} \widetilde{E}_{n} .
$$

Then $\tilde{B}=(\tilde{V}, \tilde{E})$ is a simple Bratteli diagram. For $v \in \tilde{V}_{n}$ and $\lambda \in \mathbb{R} / \mathbb{Z}$ we let $\operatorname{Var}(v, \lambda)$ be the supremum of $|m \lambda|$, where $m$ runs over all integers such that there exists $x \in X_{B}$ that goes through $v$ and whose initial $n$ edges agree with those of $\phi_{B}^{m}(x)$. Then we have the following.

Proposition 8. In the above setting,

$$
\begin{aligned}
e\left(\phi_{B}\right) & =\left\{\lambda \in \mathbb{R} / \mathbb{Z} ; \operatorname{Var}(v, \lambda) \leq 3^{-1} \text { for some } v \in \tilde{V}\right\} \\
& =\{\lambda \in \mathbb{R} / \mathbb{Z} ; \operatorname{Var}(v, \lambda) \rightarrow 0 \text { as } v \rightarrow \infty\} .
\end{aligned}
$$

Proof. Take $\lambda \in e\left(\phi_{B}\right)$. We will show that there exists a vertex $v$ such that $\operatorname{Var}(v, \lambda) \leq 3^{-1}$. For $x_{0}=\left(e_{1}, e_{2}, \ldots\right) \in X_{B} \backslash\left\{\infty_{B}\right\}$, let $U_{n}$ be a set of infinite paths whose initial $n$ edges agree with those of $x_{0}$. Then $U_{n}$ is a clopen neighborhood of $x_{0}$ and $\bigcap_{n} U_{n}=\left\{x_{0}\right\}$. Because an eigenfunction $f$ corresponding to $\lambda$ is continuous, there exists $n$ such that $|f(x)-f(y)|<3^{-1}$ for all $x, y \in U_{n}$. Then the vertex $r\left(e_{n}\right)$ does the job.

Next, suppose $\operatorname{Var}\left(w_{0}, \lambda\right) \leq 3^{-1}$ holds for some $\lambda \in \mathbb{R} / \mathbb{Z}$ and $w_{0} \in \tilde{V}_{n}$. We would like to show $\lim \operatorname{Var}(v, \lambda)=0$. By telescoping $B=(V, E)$, we may assume that all vertices $v, v^{\prime}$ lying in successive levels are connected. The proof is by contradiction. Suppose $\operatorname{Var}(v, \lambda)>\varepsilon$ holds for infinitely many $v \in \tilde{V}$. Take $k \in \mathbb{N}$ with $k \varepsilon>3^{-1}$. At first, we can find $n_{1}>n$ and $w_{1} \in \tilde{V}_{n_{1}}$ such that $\operatorname{Var}\left(w_{1}, \lambda\right)>\varepsilon$. Hence there exist an infinite path $x_{1}$ and $m_{1} \in \mathbb{Z}$ such that $x_{1}$ and $\phi^{m_{1}}\left(x_{1}\right)$ have the same initial $n_{1}$ edges and $\left|m_{1} \lambda\right|>\varepsilon$. We may assume that $x_{1}$ goes through $w_{0}$ and $m_{1} \lambda \in\left(\varepsilon, 3^{-1}\right]$ in $\mathbb{R} / \mathbb{Z}$. Since $x_{1}$ and $\phi^{m_{1}}\left(x_{1}\right)$ have the same tail, we can find $n_{2}>n_{1}$ and $w_{2} \in \tilde{V}_{n_{2}}$ such that $\operatorname{Var}\left(w_{2}, \lambda\right)$ is greater than $\varepsilon$ and the $k$-th edges of $x_{1}$ and $\phi^{m_{1}}\left(x_{1}\right)$ agree for every $k>n_{2}$. Therefore there exist an infinite path $x_{2}$ and $m_{2} \in \mathbb{Z}$, such that $x_{2}$ and $\phi^{m_{2}}\left(x_{2}\right)$ have the same initial $n_{2}$ edges and $\left|m_{2} \lambda\right|>\varepsilon$. In a similar fashion to the first step, we may assume that $x_{2}$ and $x_{1}$ have the same initial $n_{2}-1$ edges and $m_{2} \lambda \in\left(\varepsilon, 3^{-1}\right]$ in $\mathbb{R} / \mathbb{Z}$. By repeating this argument, we get $x_{k}$ and $m_{1}, m_{2}, \ldots, m_{k} \in \mathbb{Z}$. Then we have

$$
\left|\left(m_{1}+m_{2}+\cdots+m_{i}\right) \lambda\right| \leq \operatorname{Var}\left(w_{0}, \lambda\right) \leq 3^{-1}
$$

and $m_{i} \lambda \in\left(\varepsilon, 3^{-1}\right]$ for all $i=1,2, \ldots, k$, which shows a contradiction.

At last, suppose $\lim \operatorname{Var}(v, \lambda)=0$. Fix an infinite path $x_{0} \in X_{B} \backslash\left\{\infty_{B}\right\}$. We define a function $f: X_{B} \backslash\left\{\infty_{B}\right\} \rightarrow \mathbb{R} / \mathbb{Z}$ by

$$
f(x)=\lim _{n \rightarrow \infty} a_{n} \lambda
$$

when $\phi^{a_{n}}\left(x_{0}\right)$ goes to $x$. If $\phi^{i}\left(x_{0}\right)$ and $\phi^{j}\left(x_{0}\right)$ have the same initial $n$ edges $\left(e_{1}, e_{2}, \ldots, e_{n}\right)$ and $r\left(e_{n}\right)=v \in \tilde{V}_{n}$, then $|(i-j) \lambda| \leq \operatorname{Var}(v, \lambda)$. Hence the function 
$f$ is well-defined. By the same reason we have the continuity of $f$, and so $f$ is in $E(\phi)$ and $\lambda \in e(\phi)$.

We can say the following regarding the topology of $e(\phi)$.

Lemma 9. Let $B=(V, E)$ be a properly ordered almost simple Bratteli diagram as above.

(i) Suppose $\left\{\lambda_{n}\right\}_{n \in \mathbb{N}} \subset e(\phi)$ and $\lambda \in e(\phi)$. If $\lambda_{n}$ goes to $\lambda$ in $e(\phi)$, then $\operatorname{Var}\left(v, \lambda_{n}\right)$ converges to zero uniformly on $n$ as $v \rightarrow \infty$.

(ii) Suppose $\left\{\lambda_{n}\right\}_{n \in \mathbb{N}} \subset e(\phi)$ and $\lambda_{n}$ goes to $\lambda$ in $\mathbb{R} / \mathbb{Z}$. If $\operatorname{Var}\left(v, \lambda_{n}\right)$ converges to zero uniformly on $n$ as $v \rightarrow \infty$, then $\lambda$ is an eigenvalue of $\phi$ and $\lambda_{n} \rightarrow \lambda$ in $e(\phi)$.

(iii) The spectrum $e(\phi)$ is $\sigma$-compact in $\mathbb{R} / \mathbb{Z}$.

Proof. We have $\lambda_{n} \rightarrow 0$ in $e(\phi)$ if and only if $\lambda_{n} \rightarrow 0$ in $\mathbb{R} / \mathbb{Z}$ and $\sup _{v \in \tilde{V}} \operatorname{Var}\left(v, \lambda_{n}\right)$ $\rightarrow 0$ as $n \rightarrow \infty$. Thus (i) and (ii) are clear.

We prove (iii). It suffices to show that

$$
\left\{\lambda \in \mathbb{R} / \mathbb{Z} ; \operatorname{Var}(v, \lambda) \leq 3^{-1}\right\}
$$

is compact for every $v \in \tilde{V}$. But it is obvious.

Note that $e(\phi)$ itself is not $\sigma$-compact unless it is countable.

Example 10. We define an almost simple Bratteli diagram $B=(V, E)$ by the following figure.

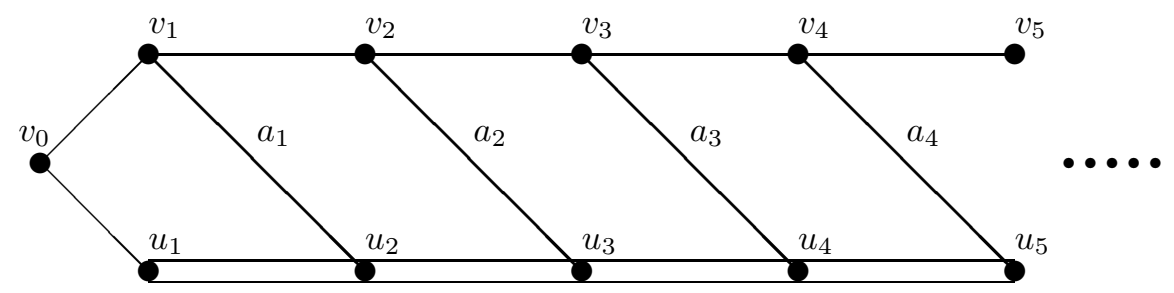

The oblique thick lines show that there exist $a_{n}$ edges from $v_{n}$ to $u_{n+1}$ for each $n \in \mathbb{N}$, where $a_{n}$ is a natural number greater than one. We introduce a linear order on each $r^{-1}\left(u_{n}\right)$ for $n \geq 2$ so that the source vertex of the maximum edge and the minimum edge is $v_{n-1}$ and the two edges from $u_{n-1}$ appear consecutively. Then $B=(V, E)$ is properly ordered.

Let $b_{n}$ be the number of paths from $v_{0}$ to $u_{n}$. Thus $\left\{b_{n}\right\}_{n}$ satisfies $b_{n+1}=$ $2 b_{n}+a_{n}$. Then, from Proposition 8 , we have

$$
e\left(\phi_{B}\right)=\left\{\lambda \in \mathbb{R} / \mathbb{Z} ; \sum_{n \in \mathbb{N}}\left|b_{n} \lambda\right|<\infty\right\} .
$$

This kind of group is called an $H_{1}$-group ([HMP]). The Polish topology of $e\left(\phi_{B}\right)$ is given by the norm $\lambda \mapsto|\lambda|+\sum\left|b_{n} \lambda\right|$.

We would like to discuss the topology of $\varepsilon(\phi)$ and duality. We have two candidates for the topology on $\varepsilon(\phi)$. One is the topology of pointwise convergence and the other is of uniform convergence on compact sets of $e(\phi)$. We denote $\varepsilon(\phi)$ 
equipped with the former weak topology by $\varepsilon(\phi)_{w}$, and denote $\varepsilon(\phi)$ equipped with the latter strong topology by $\varepsilon(\phi)_{s}$. They are obviously topological groups.

Lemma 11. For a CM or LCCM system $(X, \phi)$, the natural map $X \ni x \mapsto \tau_{x} \in$ $\varepsilon(\phi)_{s}$ is continuous. Hence $X \ni x \mapsto \tau_{x} \in \varepsilon(\phi)_{w}$ is also continuous.

Proof. Let $K \subset e(\phi)$ be a compact set. Since $P_{x_{0}}(K)$ is a compact subset of $E(\phi)$, it is equicontinuous on compact sets of $X$. Thus we have the conclusion.

For $\lambda \in e(\phi)$, we denote the homomorphism $\varepsilon(\phi) \ni \tau \mapsto \tau(\lambda) \in \mathbb{R} / \mathbb{Z}$ by $\hat{\lambda}$. Thus, $\hat{\lambda}$ is an element of the dual group of $\varepsilon(\phi)_{w}$, which is clearly a subgroup of the dual group of $\varepsilon(\phi)_{s}$. We write these dual groups by $\hat{\varepsilon}(\phi)_{w}$ and $\hat{\varepsilon}(\phi)_{s}$. The character group $\varepsilon(\phi)$ clearly contains $\mathbb{Z}$ generated by the canonical inclusion map $e(\phi) \rightarrow \mathbb{R} / \mathbb{Z}$ unless $e(\phi)$ is finite. One can show that $\mathbb{Z}$ is dense in $\varepsilon(\phi)_{w}$, and so $e(\phi)$ is isomorphic to $\hat{\varepsilon}(\phi)_{w}$ via the map $\lambda \mapsto \hat{\lambda}$ as an abelian group.

Lemma 12. In the above setting, $\lambda \mapsto \hat{\lambda}$ gives an isomorphism of topological groups $e(\phi)$ and $\hat{\varepsilon}(\phi)_{w}$, where $\hat{\varepsilon}(\phi)_{w}$ is equipped with the topology of uniform convergence on compact sets of $\varepsilon(\phi)_{s}$.

Proof. Suppose $\lambda_{n} \rightarrow 0$ in $e(\phi)$. Let $K$ be a compact subset of $\varepsilon(\phi)_{s}$. We would like to prove that $\sup _{\tau \in K} \tau\left(\lambda_{n}\right)$ goes to zero. Assume the assertion were false. Then we would have $\delta>0$ and $\tau_{n} \in K$ with $\left|\tau_{n}\left(\lambda_{n}\right)\right|>\delta$. Because $K$ is compact, we can find $\tau \in K$ whose every neighborhood contains infinitely many $\tau_{n}$. There exists a natural number $M$ such that $\left|\tau\left(\lambda_{m}\right)\right|<\delta / 4$ for all $m \geq M$. Since $\left\{\lambda_{m}\right\}_{m \geq M} \cup\{0\}$ is compact, there exists a neighborhood $U$ of $\tau$ such that $\left|\tau^{\prime}\left(\lambda_{m}\right)\right|$ is less than $\delta / 2$ for all $\tau^{\prime} \in U$. This is a contradiction.

Conversely, suppose that $\hat{\lambda}_{n}$ goes to zero in $\hat{\varepsilon}(\phi)_{w}$. Let $K$ be a compact subset of $X$. Then,

$$
\sup _{x \in K} P_{x_{0}}\left(\lambda_{n}\right)(x)=\sup _{x \in K} \hat{\lambda}_{n}\left(\tau_{x}\right) \rightarrow 0,
$$

because $\left\{\tau_{x} ; x \in K\right\}$ is compact in $\varepsilon(\phi)_{s}$.

It is unclear whether or not the canonical subgroup $\mathbb{Z}$ is dense in $\varepsilon(\phi)_{s}$. If it is dense, one can see that $\hat{\varepsilon}(\phi)_{w}$ equals $\hat{\varepsilon}(\phi)_{s}$ and the duality theorem holds.

In the above lemma, we equipped $\hat{\varepsilon}(\phi)_{w}$ with the topology of uniform convergence on compact sets of $\varepsilon(\phi)_{s}$. It is easy to see that this topology does not coincide with the topology of pointwise convergence in general. But we know the following.

Lemma 13. Let $\left\{f_{n}\right\}_{n \in \mathbb{N}} \subset E(\phi)$ be a sequence of eigenfunctions. Suppose there exists an eigenfunction $f \in E(\phi)$ such that $f_{n}(x)$ converges to $f(x)$ for all $x \in X$. Then, $f_{n}$ converges to $f$ in $E(\phi)$.

Proof. It suffices to show that $f_{n}$ converges to $f$ uniformly on compact sets of $X$. We may suppose $f=0$. Let $\lambda_{n} \in e(\phi)$ be the eigenvalue corresponding to $f_{n}$. It is clear that $\lambda_{n}$ goes to zero in $\mathbb{R} / \mathbb{Z}$.

Assume the assertion were false. Then we would have $\delta>0$ and $\left\{x_{n}\right\}_{n \in \mathbb{N}} \subset X$ such that $\left|f_{n}\left(x_{n}\right)\right|$ is greater than $\delta$ and $x_{n}$ goes to $x_{0} \in X$. At first, we can find a compact open subset $V_{1}$ such that $\left|f_{1}(y)\right|>\delta / 2$ for all $y \in V_{1}$. Put $g_{1}=f_{1}$. The minimality implies $\phi^{k}\left(x_{0}\right) \in V_{1}$ for some $k \in \mathbb{Z}$. By taking sufficiently large $m$, we may assume $\left|k \lambda_{m}\right|<\delta / 2$ and $\phi^{k}\left(x_{m}\right) \in V_{1}$. Since $\left|f_{m}\left(\phi^{k}\left(x_{m}\right)\right)\right|>\delta-\delta / 2=\delta / 2$, there exists a clopen subset $V_{2} \subset V_{1}$ such that $\left|f_{m}(y)\right|>\delta / 2$ for all $y \in V_{2}$. Put $g_{2}=$ $f_{m}$. By repeating this procedure, we will find a subsequence $\left\{g_{n}\right\}_{n}$ and a decreasing 
sequence of clopen sets $V_{n}$ such that $\left|g_{n}(y)\right|>\delta / 2$ for all $y \in V_{n}$, which implies that $g_{n}(x)$ does not converge to zero for $x \in \bigcap V_{n}$. This is a contradiction.

\section{HAUSDORFF DIMENSION}

We will prove Theorem [15] in this section. Let $D=(W, F)$ be a simple Bratteli diagram. We may assume that each vertex of $W_{1}$ connects to the top vertex by a single edge and that all vertices $w, w^{\prime}$ lying in successive levels are connected by at least two edges. We will construct an almost simple Bratteli diagram $B=(V, E)$ containing $D$ as its ideal part. Put

$$
q_{n}=\max \left\{\# r^{-1}(w) ; w \in W_{n+1}\right\} .
$$

By telescoping we may assume $2^{n^{2}} \leq q_{n}$. Let $b_{n}$ be the integer satisfying $2^{b_{n}} \leq$ $q_{n}<2^{b_{n}+1}$. Take $w_{n} \in W_{n}$ with $q_{n-1}=r^{-1}\left(w_{n}\right)$. For every $n \geq 2$ and $w \in W_{n}$, we define a linear order on $r^{-1}(w)$ so that the source vertex of the maximum edge is $w_{n-1}$ and the source vertex of the minimum edge is also $w_{n-1}$. Then $D=(W, F)$ is properly ordered.

We would like to construct an almost simple Bratteli diagram $B=(V, E)$ by adding vertices and edges to $D=(W, F)$. Let $V_{0}=\left\{v_{0}\right\}$ and let $V_{n}$ be the disjoint union of a copy of $W_{n}$ and $\left\{v_{n}\right\}$ for every $n \in \mathbb{N}$. Connect $v_{n-1}$ to $v_{n}$ by a single edge for $n \in \mathbb{N}$. These edges form the infinite path $\infty_{B}$. Take a sequence $\left\{a_{n}\right\}_{n \in \mathbb{N}}$ of natural numbers satisfying $a_{1}=0$ and $a_{n+1}>a_{n}+b_{n}+n$. We define $E_{n}$ by adding to $F_{n}$ some edges going from $v_{n-1}$ to $W_{n}$, so that the number of paths from the top vertex to $w$ in $B=(V, E)$ is $p_{n}=2^{a_{n}}$ for all $w \in W_{n}$.

Take $n \geq 2$ and $w \in W_{n}$. Let $\left(f_{1}, f_{2}, \ldots, f_{m}\right)$ be the ordered list of edges of $r^{-1}(w) \cap F_{n}$ in $D=(W, F)$. We define a linear order on each $r^{-1}(w) \subset E_{n}$, so that the ordered list of edges in $B=(V, E)$ is

$$
\left(e_{1}, e_{2}, \ldots, e_{p_{n-1}}, f_{1}, f_{2}, \ldots, f_{m}, e_{1}^{\prime}, e_{2}^{\prime}, \ldots, e_{l}^{\prime}\right),
$$

where the $e_{i}$ 's and $e_{j}^{\prime}$ 's have the source vertex $v_{n-1}$ and $2^{a_{n-1}}+m 2^{a_{n-1}}+l=2^{a_{n}}$. Then, $B=(V, E)$ is clearly a properly ordered almost simple Bratteli diagram. Let $(X, \phi)$ be the associated LCCM system.

Lemma 14. In the above setting, we have

$$
e(\phi)=\left\{\lambda \in \mathbb{R} / \mathbb{Z} ; \sum_{n=1}^{\infty}\left(q_{n}-1\right)\left|p_{n} \lambda\right|<\infty\right\} .
$$

Proof. Suppose $\lambda \in e(\phi)$. By Proposition 8 , there exists a natural number $N$ such that $\operatorname{Var}\left(w_{N}, \lambda\right) \leq 3^{-1}$. Let $\left(f_{1}, f_{2}, \ldots\right)$ be the unique minimum path in $X_{D}$. We can identify $x_{0}=\left(f_{1}, f_{2}, \ldots\right)$ with a point in $X=X_{B} \backslash\left\{\infty_{B}\right\}$. Put

$$
L^{+}=\left\{n \in \mathbb{N} ; n \geq N+1 \text { and } p_{n} \lambda \in\left[0,2^{-1}\right) \text { in } \mathbb{R} / \mathbb{Z}\right\}
$$

and

$$
L^{-}=\left\{n \in \mathbb{N} ; n \geq N+1 \text { and } p_{n} \lambda \in\left[-2^{-1}, 0\right) \text { in } \mathbb{R} / \mathbb{Z}\right\} .
$$

Let $n_{1}<n_{2}<\cdots<n_{k}$ and $n_{i} \in L^{+}$. It is easy to see that $\phi^{j p_{n_{k}}}\left(x_{0}\right)$ has the same initial $N$ edges with $x_{0}$ for $j=1,2, \ldots, q_{n_{k}}-1$. Hence we have $\left(q_{n_{k}}-1\right)\left|p_{n_{k}} \lambda\right| \leq$ $\operatorname{Var}\left(w_{N}, \lambda\right) \leq 3^{-1}$. Moreover, the initial $n_{k}$ edges of $\phi^{\left(q_{n_{k}}-1\right) p_{n_{k}}}\left(x_{0}\right)$ agree with those of $x_{0}$. Therefore $\phi^{\left(q_{n_{k}}-1\right) p_{n_{k}}+j p_{n_{k-1}}}\left(x_{0}\right)$ also has the same initial $N$ edges with $x_{0}$ for $j=1,2, \ldots, q_{n_{k-1}}-1$, and so we get

$$
\left(q_{n_{k}}-1\right)\left|p_{n_{k}} \lambda\right|+\left(q_{n_{k-1}}-1\right)\left|p_{n_{k-1}} \lambda\right| \leq 3^{-1}
$$


since $n_{k}, n_{k-1} \in L^{+}$. By repeating this argument,

$$
\sum_{i=1}^{k}\left(q_{n_{i}}-1\right)\left|p_{n_{i}} \lambda\right| \leq 3^{-1}
$$

is obtained. Hence

$$
\sum_{n \in L^{+}}\left(q_{n}-1\right)\left|p_{n} \lambda\right|+\sum_{n \in L^{-}}\left(q_{n}-1\right)\left|p_{n} \lambda\right| \leq 3^{-1}+3^{-1}=\frac{2}{3},
$$

which means that $\sum_{n}\left(q_{n}-1\right)\left|p_{n} \lambda\right|$ is finite.

Let us prove the other inclusion. We may suppose that $\sum_{n=N}^{\infty}\left(q_{n}-1\right)\left|p_{n} \lambda\right| \leq$ $6^{-1}$. We would like to prove $\operatorname{Var}\left(w_{N}, \lambda\right) \leq 3^{-1}$. To do that, it suffices to show that $|m \lambda| \leq 6^{-1}$ whenever $x_{0}$ and $\phi^{m}\left(x_{0}\right)$ have the same initial $N$ edges. There exists a natural number $M$ greater than $N$ such that the $n$th edges of $x_{0}$ and $\phi^{m}\left(x_{0}\right)$ coincide for every $n>M$. We can find $k_{M-1}$ with $0 \leq k_{M-1}<q_{M-1}$ so that the $M$-th edges of $\phi^{m}\left(x_{0}\right)$ and $\phi^{k_{M-1} p_{M-1}}\left(x_{0}\right)$ coincide. Next, we can find $k_{M-2}$ with $0 \leq k_{M-2}<q_{M-2}$ so that the $(M-1)$-st edges of $\phi^{m}\left(x_{0}\right)$ and $\phi^{k_{M-2} p_{M-2}+k_{M-1} p_{M-1}}\left(x_{0}\right)$ coincide. By repeating this procedure, we get

$$
m=k_{N} p_{N}+k_{N+1} p_{N+1}+\cdots+k_{M-1} p_{M-1}
$$

for some $0 \leq k_{i}<q_{i}$. Therefore $|m \lambda| \leq 6^{-1}$.

Since $2^{b_{n}} \leq q_{n}<2^{b_{n}+1}$,

$$
e(\phi)=\left\{\lambda \in \mathbb{R} / \mathbb{Z} ; \sum_{n=1}^{\infty} 2^{b_{n}}\left|2^{a_{n}} \lambda\right|<\infty\right\},
$$

where $\left\{a_{n}\right\}_{n}$ and $\left\{b_{n}\right\}_{n}$ satisfy $a_{n+1}>a_{n}+b_{n}+n$ and $b_{n} \geq 2^{n}$. Put

$$
K=\bigcup_{n=1}^{\infty}\left[a_{n}, a_{n}+b_{n}\right], L=\bigcup_{n=1}^{\infty}\left[a_{n}, a_{n}+b_{n}+n\right] .
$$

Then, from [IKS], we can conclude that the Hausdorff dimension of $e(\phi)$ equals

$$
1-\limsup _{n \rightarrow \infty} \frac{1}{n} \#(K \cap[0, n])=1-\limsup _{n \rightarrow \infty} \frac{1}{n} \#(L \cap[0, n]) .
$$

Hence we obtain the following theorem.

Theorem 15. Let $\left(D, D^{+}\right)$be a simple dimension group and $\alpha \in[0,1]$ a real number. Then there exists an LCCM system $(X, \phi)$ such that the Hausdorff dimension of $e(\phi)$ is equal to $\alpha$ and $K^{0}(X, \phi)$ is isomorphic to $\left(D, D^{+}, D^{+}\right)$.

Proof. If $\alpha \neq 1$, let $a_{n}$ be the largest integer that is not greater than

$$
\frac{\sum_{i=1}^{n-1} b_{i}+i}{1-\alpha}+n
$$

If $\alpha=1$, let $a_{n}$ be a sequence increasing rapidly. 


\section{REFERENCES}

[A] Aaronson, J.; The eigenvalues of nonsingular transformations, Israel J. Math. 45 (1983), 297-312. MR 86c:28041

[AN] Aaronson, J.; Nadkarni, M.; $L_{\infty}$ eigenvalues and $L_{2}$ spectra of nonsingular transformations, Proc. London Math. Soc. (3) 55 (1987), 538-570. MR 88j:28012

[D] Danilenko, A. I.; Strong orbit equivalence of locally compact Cantor minimal systems, Internat. J. Math. 12 (2001), 113-123. MR 2002j:37016

[GPS] Giordano, T.; Putnam, I. F.; Skau, C. F.; Topological orbit equivalence and $C^{*}$-crossed products, J. reine angew. Math. 469 (1995), 51-111. MR 97g:46085

[HMP] Host, B.; Mela, J.-F.; Parreau, F.; Nonsingular transformations and spectral analysis of measures, Bull. Soc. Math. France 119 (1991), 33-90. MR 93d:43002

[IKS] Ito, Y.; Kamae, T.; Shiokawa, I.; Point spectrum and Hausdorff dimension, Number theory and combinatorics (Japan 1984), 209-227, World Sci. Publishing, Singapore, 1985. MR 87h:28018

[M] Matui, H.; Topological orbit equivalence of locally compact Cantor minimal systems, Ergodic Theory Dynam. Systems 22 (2002), 1871-1903.

[S] Srivastava, S. M.; A course on Borel sets, Graduate Texts in Mathematics 180, SpringerVerlag, New York, 1998. MR 99d:04002

Department of Mathematics and Informatics, Faculty of Science, Chiba University, YAyoityô 1-33, InAGeKU, Chiba, 263-8522, JAPAN

E-mail address: matui@math.s.chiba-u.ac.jp 